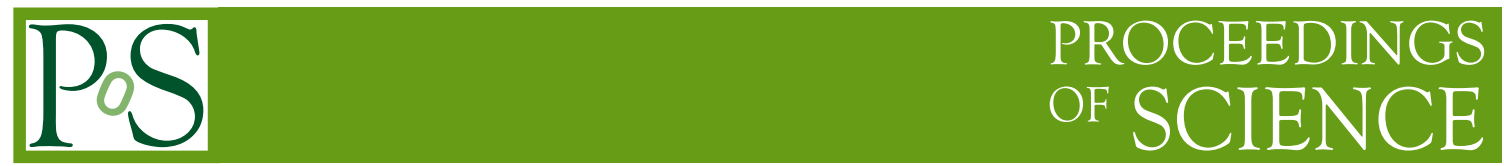

\title{
Neutrino Oscillations and Light Dark Sectors
}

\author{
Luca Vecchi* \\ Theoretical Particle Physics Laboratory, Institute of Physics, EPFL, Lausanne, Switzerland \\ E-mail: luca.vecchi@epfl.ch
}

In this note we argue that, due to a coherent enhancement, neutrino oscillation experiments are sensitive to very feeble interactions between the Standard Model and dark sectors, as long as these are sufficiently light. This offers a unique probe of light new physics sectors coupled to us via the neutrino portal.

Neutrino Oscillation Workshop (NOW2018)

9 - 16 September, 2018

Rosa Marina (Ostuni, Brindisi, Italy)

\footnotetext{
${ }^{*}$ Speaker.
} 


\section{The framework}

Suppose the dark world (i.e. dark matter, dark energy, dark radiation) possesses a small coupling to the Standard Model (SM) neutrinos. This can be achieved introducing a family of fermion singlets (i.e. right-handed neutrinos) $N_{a}$ that couple to the dark sector (say via the exchange of a scalar or a vector boson) as well as the SM lepton $\left(\ell_{i}=\left(v_{\alpha}, e_{\alpha}\right)^{t}\right)$ and Higgs $(H)$ doublets via

$$
\delta \mathscr{L}=y_{a \alpha} N_{a} H \ell_{\alpha}+\text { hc } .
$$

In this picture the right-handed neutrinos are messengers that mediate an interaction between the SM and the dark world. After electroweak symmetry breaking $v_{\alpha}$ and $N_{a}$ mix with an angle $\sim \theta$ and so the active neutrinos effectively inherit a coupling to the dark sector.

Now it becomes possible to have reactions such as $v+X \leftrightarrow v+X^{\prime}, v+X \leftrightarrow N+X^{\prime}$, where $X, X^{\prime}$ are particles in the dark sector. At large momentum transfers $q$ these have cross sections that scale as $g^{4} \theta^{4} / q^{2}, g^{4} \theta^{2} / q^{2}$, with $g$ the coupling between $N$ and the dark particles. As usual, nothing exotic can be observed in the decoupling limit $g^{2} \rightarrow 0$.

The situation is qualitatively different at small momentum transfer if the particles $X$ have a non-vanishing background within which the active neutrinos propagate, which is precisely the situation when $X$ constitutes part or the entirety of the missing energy in our universe. In that case a single active neutrino may interact with a whole distribution of $X$ particles and the processes

$$
v+X \leftrightarrow v+X
$$

can receive a coherent enhancement. The net effect can be described in terms of a modification of the dispersion relation of the active neutrinos, the energy of which receive a correction of order $\delta V_{\text {eff }} \sim\left(g^{2} / m^{2}\right) \sin ^{2} \theta n_{X}$, where $n_{X}$ is the number density of the background $X$ particles and $m$ some IR cutoff. Of course a completely analogous situation occurs when the SM neutrinos traverse ordinary matter and $\theta$ represents an active/active mixing angle, $g^{2} / \mathrm{m}^{2}$ the Fermi constant, and $n_{X}$, say, the number density of electrons. The crucial difference is that in our case the new effect involves unknown forms of matter, with unknown couplings and masses. In particular, it may even be possible to have sizable effects with $g^{2} \ll 1, m^{2} \rightarrow 0$ as long as $g^{2} / m^{2}$ is kept fixed: extremely weakly-coupled new physics may result in appreciable deviations of the neutrino properties if the dark sector is sufficiently light! Crucially, only neutrinos — being largely unaffected by the environment otherwise - can be sensitive to such coherent enhancements. In this sense neutrino oscillation experiments represent a unique opportunity to probe light dark sectors.

The dark background might be due to radiation (in which case the typical expectation is $n_{X} \sim$ $\left.1 / \mathrm{cm}^{3}\right)$ or non-relativistic matter $\left(n_{X}=\rho_{X} / m_{X}\right.$, with $\left.\rho_{X} \sim 0.4 \mathrm{GeV} / \mathrm{cm}^{3}\right)$. A simple estimate gives

$$
\delta V_{\text {eff }} \sim \frac{g^{2}}{m^{2}} \sin ^{2} \theta n_{X} \sim 10^{-23} \mathrm{GeV}\left(\frac{\sin \theta}{10^{-3}}\right)^{2} \begin{cases}\left(\frac{\mathrm{meV}}{m / g}\right)^{2} \frac{n_{X}}{1 / \mathrm{cm}^{3}}, & \text { dark radiation } \\ \left(\frac{\mathrm{eV}}{m / g}\right)^{2} \frac{\rho_{X}}{0.4 \mathrm{GeV} / \mathrm{cm}^{3}} \frac{1 \mathrm{keV}}{m_{X}}, & \text { dark matter. }\end{cases}
$$

To observe an effect of this type in neutrino oscillation experiments the mixing $\theta$ should be flavorviolating, otherwise all neutrinos would be equally affected. This is in fact expected to be the case with a generic $y_{a \alpha}$ in (1.1). Now the question becomes: What is the reach of present and future experiments on a flavor-violating $\delta V_{\text {eff }}$ ? What is the most promising technique to probe it? 


\section{Results}

In [1] we considered the most general Lagrangian quadratic in the SM neutrinos and found that at leading order in the new physics corrections, the dispersion relation of relativistic active neutrinos is affected linearly by two 3 by 3 matrices $a, b$. The resulting effective 6-dimensional Hamiltonian (involving neutrinos and anti-neutrinos) reads $H_{\text {eff }}=H_{\mathrm{SM}}+\delta V_{\text {eff }}$, with

$$
H_{\mathrm{SM}}=\left(\begin{array}{cc}
U \frac{m_{v} m_{v}^{*}}{2 E} U^{\dagger} & \\
& U^{*} \frac{m_{v}^{*} m_{v}}{2 E} U^{t}
\end{array}\right) \quad \delta V_{\mathrm{eff}}=\left(\begin{array}{cc}
a(\hat{\mathbf{p}}) & b(\hat{\mathbf{p}}) \\
b^{\dagger}(\hat{\mathbf{p}}) & -a^{*}(\hat{\mathbf{p}})
\end{array}\right),
$$

where $a(\hat{\mathbf{p}})$ and $b(\hat{\mathbf{p}})$ are respectively hermitian and anti-symmetric in the flavor indices.

The future reach on $\delta V_{\text {eff }}$ from atmospheric data from the Orca experiment and IceCube after 10 years of data taking are estimated as in the figure below (obtained switching on one coupling at a time). For details see [1]. Comparing to (1.3) we find that future atmospheric neutrino experiments are sensitive to dark sectors with scales in the $g^{2} / m^{2} \sim \mathrm{meV}^{2}-\mathrm{keV}^{2} \gg G_{F}$ range.
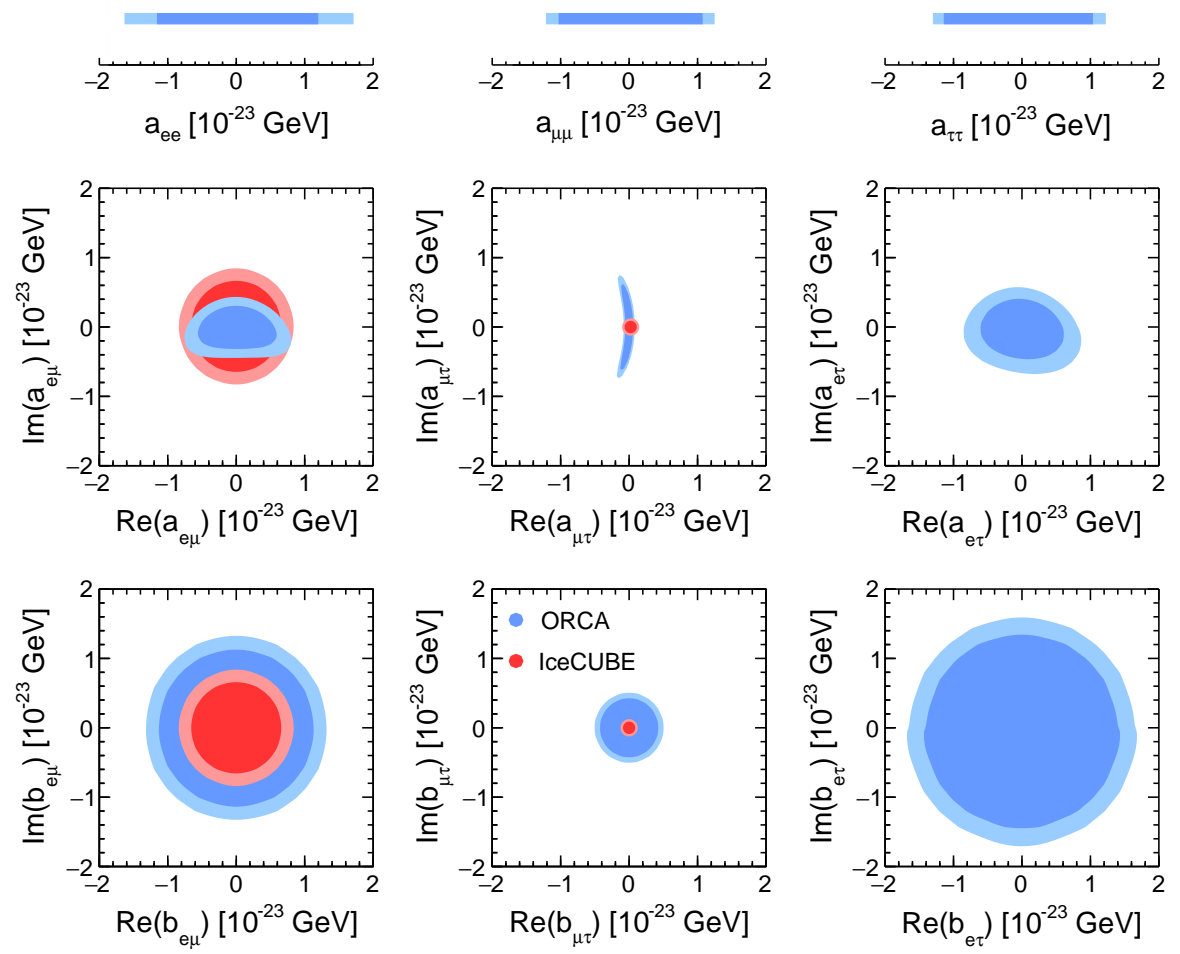

Figure 1: Projected 10 year sensitivities at IceCube (high-energy and muon only) and ORCA to dark backgrounds with nonzero $a(\hat{\mathbf{p}})$ and $b(\hat{\mathbf{p}})$ couplings each turned on one at a time. Note that $b(\hat{\mathbf{p}})$ couplings have no interference effects with the SM, which explains the circular regions. The same is true for all IceCube sensitivities which are done at energies so large that the SM contribution is negligible. These bounds assume normal hierarchy.

All information on the actual size of $\mathrm{m}^{2} / \mathrm{g}^{2}$ would however be lost if we employed very energetic neutrinos (e.g. astrophysical neutrinos). In this latter case neutrino oscillations would be 
completely controlled by $\delta V_{\text {eff }}$, with no interference with $H_{\mathrm{SM}}$, and only relative angles within the various components in $\delta V_{\text {eff }}$ could be observed. In order not to miss this important information, and to simultaneously maximize the reach it is crucial to use atmospheric data [1].

It should also be stressed that, while here we assumed $\delta V_{\text {eff }}$ provides a subleading correction to the SM, neutrino oscillations themselves may originate from the coupling between active neutrinos and dark backgrounds, even in the absence of electroweak symmetry breaking (i.e. $m_{v}=0$ ). [1]

\section{References}

[1] F. Capozzi, I. M. Shoemaker and L. Vecchi, JCAP 1807, no. 07, 004 (2018) doi:10.1088/1475-7516/2018/07/004 [arXiv:1804.05117 [hep-ph]]. 